\title{
ESTUDO DAS DIFERENTES FORMAS DE FÓSFORO NAS ÁGUAS DA PLATAFORMA CONTINENTAL DO AMAZONAS
}

\author{
Maria de Lourdes Souza Santos* \\ Universidade Federal do Pará, Alameda Leandro Ribeiro, s/n, 68600-000 Bragança - PA, Brasil \\ Kátia Muniz e Fernando Antonio do Nascimento Feitosa \\ Departamento de Oceanografia, Universidade Federal de Pernambuco, Av. Arquitetura, s/n, 50740-550 Recife - PE, Brasil \\ Benício de Barros Neto \\ Departamento de Química Fundamental, Universidade Federal de Pernambuco, Av. Arquitetura, s/n, 50740-550 Recife - PE, Brasil
}

Recebido em 14/2/06; aceito em 26/7/06; publicado na web em 19/1/07

\begin{abstract}
STUDY OF DIFFERENT FORMS OF PHOSPHORUS IN THE AMAZON CONTINENTAL SHELF WATERS. Hydrological samples were collected on the continental shelf of the Amazon river to determine particulate organic and inorganic matter, dissolved organic phosphorus (DOP), phosphate, total particulate phosphorus (TPP), chlorophyll $a$, temperature, salinity, $\mathrm{pH}$, water transparency, dissolved oxygen (DO) and saturation rate. The objective was to study the forms of DOP, phosphate and TPP. In the euphotic layer, the fluvial discharge from the Amazon river favored the distribution of phosphate, TPP and DOP. In the aphotic layer, the phosphate concentration increased due to the decomposition of DOP and TPP. This increase is due to the absence of photosynthetic processes.
\end{abstract}

Keywords: Amazon; phosphorus; chlorophyll $a$.

\section{INTRODUÇÃO}

O fósforo transportado através das águas dos rios para os oceanos passa pelos estuários onde consideráveis processos ocorrem, alterando a disponibilidade biológica e o fluxo deste elemento para as áreas costeira e oceânica. O fósforo é assimilado pelo fitoplâncton, bactérias, e plantas bênticas, e é remineralizado por atividades heterotróficas dos animais e microorganismos. Por outro lado, a mistura da água do rio com a água do mar, nos estuários cria algumas características químicas únicas, que afetam a adsorção do fosfato inorgânico nas partículas.

Berner e Rao ${ }^{1}$ destacaram a importância do estudo do fósforo transportado pelo rio Amazonas para o oceano, devido à magnitude de descarga de água e por ser um rio relativamente não poluído. O volume de água doce desse rio ocasiona alterações nos parâmetros hidrológicos locais. A descarga hídrica do rio Amazonas é da ordem de 100.000 a $220.000 \mathrm{~m}^{3} \mathrm{~s}^{-1}$, e a descarga sólida é de 11 a $13 \mathrm{x}$ $10^{8}$ t por ano $^{2}$.

Fox et al. ${ }^{3}$, Fox $^{4}$, Berner e Rao ${ }^{1}$ examinaram a sistemática do fosfato no Amazonas, e em vários outros ecossistemas, concluindo que $50 \%$ do fosfato liberado para o oceano a partir do rio Amazonas, é o resultado da dissociação dos sedimentos em suspensão, existindo um equilíbrio entre as formas particuladas e dissolvidas (principalmente o fosfato férrico/mineral hidróxido férrico), controlando a concentração de fosfato nas águas de baixa salinidade na plataforma.

O estudo do fósforo na área da Plataforma Continental do Amazonas tem sido principalmente restrito à forma inorgânica dissolvida e às diversas formas existentes nos sedimentos. Pesquisas com fósforo orgânico dissolvido (POD) e fósforo total particulado (PTP) são escassas nessa região.

Os objetivos do presente trabalho foram utilizar análise de componentes principais para relacionar todas as formas do fósforo na

\footnotetext{
*e-mail: mdelssantos@yahoo.com.br
}

coluna de água: fósforo orgânico dissolvido (POD), fosfato, fósforo total particulado (PTP) com os parâmetros abióticos (salinidade, temperatura, $\mathrm{pH}$, oxigênio dissolvido (OD), transparência da água, material particulado orgânico (MPO), material particulado inorgânico (MPI)) e clorofila $a$, a fim de determinar possíveis correlações entre esses parâmetros; analisar a distribuição das diferentes formas do fósforo em relação à mistura das massas de água fluvial e salina (diagrama de mistura) e, comparar a presença de cada forma do ciclo do fósforo nas camadas eufótica e afótica, a fim de melhor visualizar a distribuição no ambiente pelágico.

\section{PARTE EXPERIMENTAL}

Os dados foram obtidos no período de transição das águas do rio Amazonas (entre as descargas máxima e mínima), durante a realização da Operação Norte IV (2001), a bordo do Navio Oceanográfico "ANTARES", pertencente à Marinha do Brasil, dentro do âmbito do Programa "Avaliação do Potencial Sustentável de Recursos Vivos na Zona Econômica Exclusiva"- REVIZEE.

Foram utilizados os dados obtidos na coluna de água nas estações localizadas na área da Plataforma Continental do Amazonas (agosto de 2001), distribuídas ao longo de 11 perfis, totalizando 41 estações (Figura 1).

As amostras de água foram coletadas utilizando garrafas de Niskin de $5 \mathrm{~L}$. Os seguintes dados foram obtidos a bordo do navio: temperatura e salinidade registrados por um CTD ("Conductivity, Temperature, Depth") SeaBird SBE 9-plus ligado à unidade de bordo SBE 11 acoplado a uma Rosette; transparência da água utilizando o disco de Secchi (m); oxigênio dissolvido utilizando-se o método de Winkler, descrito em Strickland e Parsons ${ }^{5}$; taxa de saturação do oxigênio dissolvido, utilizando a "International Oceanographic Tables"6 e, pH medido com um pHmetro da marca Hanna.

Para determinação do fósforo total dissolvido e do fosfato as amostras de água foram filtradas em filtros de $0,45 \mu \mathrm{m} \mathrm{GF/F} \mathrm{da}$ marca Whatman, armazenadas em frascos de polietileno de 500 $\mathrm{mL}$ e congeladas. Para a determinação do fósforo total, as amos- 


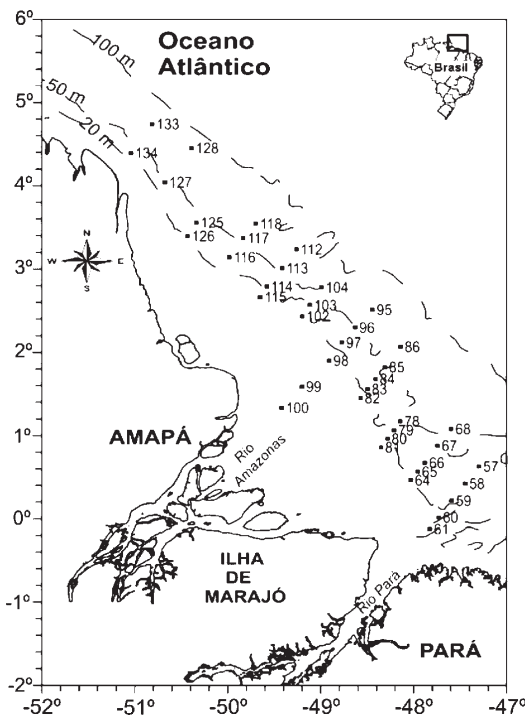

Figura 1. Localização das estações oceanográficas (Programa REVIZEE/ Operação Norte IV)

tras de água foram apenas armazenadas em frascos de polietileno de $250 \mathrm{~mL}$, sem passar pelo processo de filtração.

Posteriormente, no Laboratório de Oceanografia da Universidade Federal do Pará, as amostras de água foram descongeladas e as análises feitas em duplicatas para determinações de: fósforo total dissolvido, fósforo total, fosfato, aplicando-se a metodologia descrita em Grasshoff et al. ${ }^{7}$. No laboratório a água utilizada para determinação era proveniente de um ALPHA Q.

Para obter a concentração do fósforo total particulado, foram feitas as análises do fósforo total (amostra não-filtrada) e fósforo total dissolvido (amostra filtrada) por meio do método da oxidação por via úmida com persulfato de potássio, em meio básico. A diferença entre fósforo total e fósforo total dissolvido dá uma estimativa do fósforo total particulado.

Por outro lado, a concentração do fósforo orgânico dissolvido é estimada por meio das análises feitas com as amostras filtradas, pela diferença entre fósforo total dissolvido e fosfato.

Após a oxidação das amostras o principio do método é o mesmo utilizado para o fosfato. Ou seja, dosar o fósforo na forma de íon ortofosfato, os quais reagem em meio ácido com o molibdato de amônio, formando o complexo fosfomolibdato. Este complexo é então reduzido pelo ácido ascórbido, numa reação catalisada pelo antimônio tartarato de potássio, formando um complexo de coloração azul que tem absorção máxima em $880 \mathrm{~nm}$. A leitura foi feita em um espectrofotômetro da marca QUIMIS. Segundo Paranhos ${ }^{8}$ a precisão desse método pode ser considerada como sendo de $\pm 15 \%$ na faixa de concentração de $0,2 \mu \mathrm{mol} \mathrm{L} \mathrm{L}^{-1}$ de fosfato.

O material particulado orgânico e inorgânico foram determinados pelo método gravimétrico descrito em Paranhos ${ }^{8}$. Para clorofila $a$, as análises foram feitas no Laboratório de Produtividade Primária do Departamento de Oceanografia da Universidade Federal de Pernambuco, utilizando-se o método descrito por Teixeira ${ }^{9}$.

Os pigmentos são quantificados por meio de sua absorção de luz em diferentes comprimentos de onda. Os filtros foram colocados em tubos de centrifuga, adicionados $10 \mathrm{~mL}$ de acetona $90 \%$. Após $24 \mathrm{~h}$ foram centrifugados por $10 \mathrm{~min}$ em $4.000 \mathrm{rpm}$. O sobrenadante foi transferido para a cubeta do espectrofotômetro. E leituras foram feitas nos seguintes comprimentos de onda: 750 , $665,664,647,630,510$ e $480 \mathrm{~nm}$.

Os dados de salinidade, temperatura, $\mathrm{pH}$, oxigênio dissolvido (OD), taxa de saturação de oxigênio dissolvido, material particulado orgânico (MPO), material particulado inorgânico (MPI), fósforo orgânico dissolvido (POD), fosfato, fósforo total particulado (PTP) e clorofila $a$ obtidos foram analisados utilizando-se métodos de estatística descritiva e de análise de componentes principais.

\section{RESULTADOS E DISCUSSÃO}

Inicialmente foram feitas análises de componentes principais para as camadas eufótica e afótica. Na camada eufótica, as duas primeiras componentes (PC1 e PC2) explicaram 52,4\% da variância original, com $29,4 \%$ para a primeira componente e $23 \%$ para a segunda componente (Tabela 1).

Tabela 1. Pesos e variância explicada pelas duas primeiras componentes principais da análise dos dados da camada eufótica obtidos no período de transição do rio Amazonas, na Plataforma Continental do Amazonas. Os valores mais significativos estão em negrito

\begin{tabular}{lcc}
\hline Variável & PC1 & PC2 \\
\hline Temperatura $\left({ }^{\circ} \mathrm{C}\right)$ & $-0,19$ & $\mathbf{- 0 , 7 4}$ \\
Salinidade & $\mathbf{- 0 , 6 3}$ & $\mathbf{0 , 6 4}$ \\
MPI $\left(\mathrm{mg} \mathrm{L}^{-1}\right)$ & 0,08 & $-0,18$ \\
MPO $\left(\mathrm{mg} \mathrm{L}^{-1}\right)$ & $\mathbf{0 , 6 2}$ & 0,32 \\
pH & $\mathbf{- 0 , 4 9}$ & $-0,25$ \\
Clorofila $a\left(\mathrm{mg} \mathrm{m}^{-3}\right)$ & $\mathbf{- 0 , 4 9}$ & $\mathbf{- 0 , 5 9}$ \\
OD $\left(\mathrm{mL} \mathrm{L}^{-1}\right)$ & $\mathbf{- 0 , 4 5}$ & $\mathbf{- 0 , 6 6}$ \\
Fosfato $\left(\mu \mathrm{mol} \mathrm{L}^{-1}\right)$ & $\mathbf{0 , 8 4}$ & $-0,15$ \\
PTP $\left(\mu \mathrm{mol} \mathrm{L}^{-1}\right)$ & $\mathbf{0 , 7 4}$ & $\mathbf{- 0 , 4 8}$ \\
POD $\left(\mu \mathrm{mol} \mathrm{L}^{-1}\right)$ & 0,44 & $-0,32$ \\
Variância explicada $(\%)$ & 29,4 & 23 \\
\hline
\end{tabular}

$\mathrm{Na}$ primeira componente, foi observado um contraste entre o MPO $(0,62)$, fosfato $(0,84)$, PTP $(0,74)$, POD $(0,44)$, e a salinidade $(-0,63), \mathrm{pH}(-0,49)$, Clorofila $a(-0,49)$, OD $(-0,45)$. Esta correlação negativa sugere que a concentração de clorofila $a$ foi favorecida pela disponibilidade do fosfato, aumentando conseqüentemente o pH e o OD, em áreas com salinidade elevada. A correlação positiva entre o PTP, POD e fosfato demonstra a ligação existente entre as diversas formas do fósforo, com a transformação do PTP para o POD ou fosfato, e também do POD para o fosfato. A relação inversa desses parâmetros com o oxigênio dissolvido e com a salinidade, ratifica o processo de transformação entre essas formas e o aporte oriundo das águas do rio Amazonas, respectivamente. A correlação negativa entre a salinidade e o MPO demonstra que a concentração do material particulado orgânico está sendo favorecida pelas águas oriundas do rio Amazonas.

A segunda componente apresenta como principais elementos à temperatura $(-0,74)$, Clorofila $a(-0,59)$, OD $(-0,66)$, PTP $(-0,48)$, em contraste com a salinidade $(0,64)$, mostrando a influência da água fluvial na distribuição de Clorofila $a$, e conseqüente aumento de OD e do PTP, em águas com temperaturas significativas.

A Figura 2 apresenta os escores nas duas primeiras componentes principais, estratificados de acordo com as profundidades de coleta. A coluna de água apresenta-se bastante misturada, entre a superfície e a camada considerada até $1 \%$ de luz. Porém, com pequena separação entre a superfície e a camada considerada até $1 \%$ de luz na PC2. Os dados de águas de superfície ao longo da PC2 apresentaram escores mais negativos, indicando maiores valores de temperatura, OD e Clorofila $a$, enquanto na camada considerada até $1 \%$ de luz os escores mais positivos indicam maiores valores de salinidade. Na PC1 não se observa uma separação nítida, mas as estações 133 e 99 se destacam das demais. A primeira, 
devido ao elevado valor de OD e clorofila $a$ e a segunda, as elevadas concentrações de fosfato e de PTP.

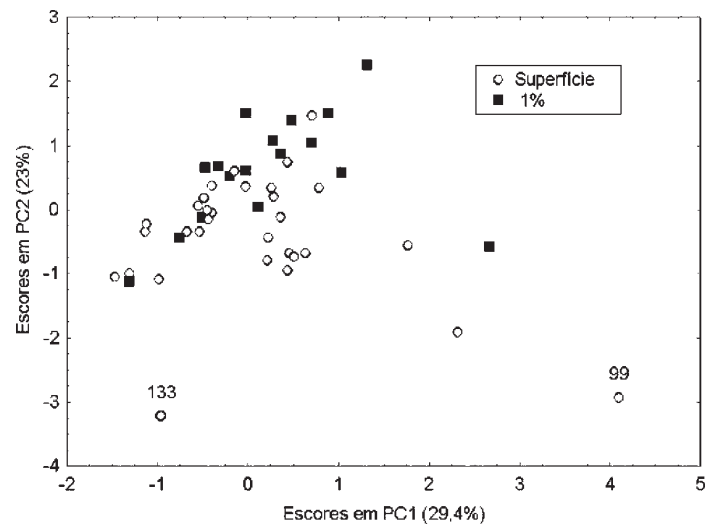

Figura 2. Escores nas duas primeiras componentes principais, na análise feita com os dados abióticos e clorofila a da camada eufótica, com estratificação entre as profundidades de coleta (superfície e $1 \%$ de penetração de luz)

Na camada afótica as duas primeiras componentes explicaram cerca de $47,5 \%$ da variância original (Tabela 2). A primeira componente explicou $27,5 \%$ e apresentou como principais elementos o $\mathrm{pH}(-0,42)$ e o OD $(-0,57)$ em contraste com o MPI $(0,66)$, MPO $(0,44)$, fosfato $(0,79)$, PTP $(0,68)$ e POD $(0,63)$. A correlação negativa do MPO, PTP, POD com OD pode ser explicada pelo processo de degradação da matéria orgânica, que libera o fosfato para a coluna de água.

Tabela 2. Pesos e variância explicada pelas duas primeiras componentes principais da análise dos dados da camada afótica obtidos no período de transição do rio Amazonas, na Plataforma Continental do Amazonas. Os valores mais significativos estão em negrito

\begin{tabular}{lcc}
\hline Variável & PC1 & PC2 \\
\hline Temperatura $\left({ }^{\circ} \mathrm{C}\right)$ & 0,07 & $\mathbf{- 0 , 8 8}$ \\
Salinidade & 0,37 & $\mathbf{0 , 6 9}$ \\
MPI $\left(\mathrm{mg} \mathrm{L}^{-1}\right)$ & $\mathbf{0 , 6 6}$ & $\mathbf{- 0 , 4 8}$ \\
MPO $\left(\mathrm{mg} \mathrm{L}^{-1}\right)$ & $\mathbf{0 , 4 4}$ & $\mathbf{0 , 5 1}$ \\
pH & $\mathbf{- 0 , 4 2}$ & $-0,36$ \\
OD $\left(\mathrm{mL} \mathrm{L}^{-1}\right)$ & $\mathbf{- 0 , 5 7}$ & $-0,01$ \\
Fosfato $\left(\mathrm{mmol} \mathrm{L}^{-1}\right)$ & $\mathbf{0 , 7 9}$ & 0,35 \\
PTP $\left(\mathrm{mmol} \mathrm{L}^{-1}\right)$ & $\mathbf{0 , 6 8}$ & 0,02 \\
POD $\left(\mathrm{mmol} \mathrm{L}^{-1}\right)$ & $\mathbf{0 , 6 3}$ & $-0,05$ \\
Variância explicada $(\%)$ & 27,5 & 20 \\
\hline
\end{tabular}

A segunda componente (PC2) explicou cerca de $20 \%$ da variância total, apresentando um contraste entre temperatura $(-0,88)$, MPI $(-0,48)$ e a salinidade $(0,69)$ e MPO $(0,51)$. A correlação positiva entre o MPO e a salinidade sugere a liberação de matéria orgânica com o aumento da profundidade.

Os valores estatísticos descritivos das distribuições do fosfato, fósforo total particulado e fósforo orgânico dissolvido encontram-se na Tabela 3. As concentrações de fosfato (camada eufótica: mínimo de 0,06 e máximo de $0,77 \mu \mathrm{mol} \mathrm{L} \mathrm{L}^{-1}$, e camada afótica: mínimo de 0,12 e máximo de $0,89 \mu \mathrm{mol} \mathrm{L}^{-1}$ ), obtidas no presente trabalho, foram baixas quando comparadas às de sistemas estuarinos que sofrem influências antropogênicas como em Hudson $(2,0-6,5 \mu \mathrm{mol}$ $\mathrm{L}^{-1}$, Clark et al..$\left.^{10}\right)$, Baía de São Francisco $\left(3,1-13,7 \mu \mathrm{mol} \mathrm{L}{ }^{-1}{ }^{11}\right)$, Tanshui $\left(0,35-5,46 \mu \mathrm{mol} \mathrm{L}{ }^{-1}{ }^{12}\right)$ e no rio Paraíba do Sul $(0,43-1,66$ $\left.\mu \mathrm{mol} \mathrm{L} \mathrm{L}^{-1}{ }^{13}\right)$. Isto permite caracterizar a área em estudo como um ambiente não impactado em relação a esse nutriente.

No ambiente marinho, o POD é considerado como o maior reservatório de fósforo e importante fonte de fósforo biologicamente dis-
Tabela 3. Estatística descritiva para o fosfato, PTP, POD, e fósforo total dissolvido (PTD) na camada eufótica e afótica, dos dados obtidos no período de transição do rio Amazonas, na Plataforma Continental do Amazonas

\begin{tabular}{|c|c|c|c|c|c|}
\hline & & $\begin{array}{c}\text { Fosfato } \\
\left.(\mu \mathrm{mol} \mathrm{L})^{-1}\right)\end{array}$ & $\begin{array}{c}\text { PTP } \\
\text { amol L'-1) }\end{array}$ & $\begin{array}{c}\text { POD } \\
\left.(\mu \mathrm{mol} \mathrm{L})^{-1}\right)\end{array}$ & $\begin{array}{c}\text { PTD } \\
\left.(\mu \mathrm{mol} \mathrm{L})^{-1}\right)\end{array}$ \\
\hline & $\mathrm{N}^{\mathrm{o}}$ de valores & 70 & 70 & 70 & 70 \\
\hline & Mínimo & 0,06 & 0,04 & 0,01 & 0,10 \\
\hline Camada & Máximo & 0,77 & 1,78 & 0,40 & 0,94 \\
\hline \multirow[t]{5}{*}{ Eufótica } & Mediana & 0,20 & 0,13 & 0,07 & 0,27 \\
\hline & Quartil superior & - 0,12 & 0,10 & 0,04 & 0,20 \\
\hline & Quartil inferior & 0,32 & 0,25 & 0,12 & 0,40 \\
\hline & $\mathrm{N}^{\mathrm{o}}$ de valores & 50 & 50 & 50 & 50 \\
\hline & Mínimo & 0,12 & 0,01 & 0,01 & 0,15 \\
\hline Camada & Máximo & 0,89 & 1,58 & 1,43 & 2,32 \\
\hline \multirow[t]{3}{*}{ Afótica } & Mediana & 0,31 & 0,13 & 0,09 & 0,37 \\
\hline & Quartil superior & - 0,20 & 0,05 & 0,04 & 0,29 \\
\hline & Quartil inferior & 0,40 & 0,31 & 0,14 & 0,50 \\
\hline
\end{tabular}

ponível, para os microorganismos auto e heterotróficos no ecossistema marinho ${ }^{14}$. No ambiente costeiro, o POD também tem sido reconhecido como uma fração significante do fósforo e como uma fonte potencial de fósforo para os organismos marinhos costeiros ${ }^{15}$.

Na Plataforma Continental do Amazonas, os valores da mediana

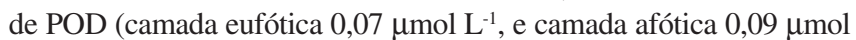
$\mathrm{L}^{-1}$ ), foram inferiores ao fosfato (camada eufótica $0,20 \mu \mathrm{mol} \mathrm{L} \mathrm{L}^{-1}$, e camada afótica $0,31 \mu \mathrm{mol} \mathrm{L^{-1 }}$ ), indicando uma pequena contribuição do POD no fósforo total dissolvido (PTD) na área em estudo, o qual apresentou mínimo de 0,10 e máximo de $0,94 \mu \mathrm{mol} \mathrm{L}^{-1}$ na camada eufótica e, mínimo de 0,15 e máximo de $2,32 \mu \mathrm{mol} \mathrm{L^{-1 }}$ na camada afótica (Tabela 3).

No entanto, em outras áreas, como em rios localizados na Geórgia, Weston et al. ${ }^{16}$ consideraram que o POD (Altamaha (0,50 $\left.\mu \mathrm{mol} \mathrm{L}{ }^{-1}\right)$, Ocmulgee $\left(0,60 \mu \mathrm{mol} \mathrm{L}{ }^{-1}\right)$, Oconee $(0,90 \mu \mathrm{mol} \mathrm{L}-1)$, Ohoopee $\left(0,60 \mu \mathrm{mol} \mathrm{L}^{-1}\right)$ e Little Ocmulgee $\left.\left(0,80 \mu \mathrm{mol} \mathrm{L}{ }^{-1}\right)\right)$ contribui de forma significativa para o reservatório de fósforo total dissolvido rios, com concentrações próximas ao fosfato (Altamaha $(0,60$ $\left.\mu \mathrm{mol} \mathrm{L} \mathrm{L}^{-1}\right)$, Ocmulgee $\left(0,70 \mu \mathrm{mol} \mathrm{L} \mathrm{L}^{-1}\right)$, Oconee $(0,80 \mu \mathrm{mol} \mathrm{L}-1)$, Ohoopee $\left(0,70 \mu \mathrm{mol} \mathrm{L}{ }^{-1}\right)$ e Little Ocmulgee $\left.\left(0,30 \mu \mathrm{mol} \mathrm{L} \mathrm{L}^{-1}\right)\right)$.

$\mathrm{Na}$ área da Plataforma Continental do Amazonas, trabalho realizado por Fox et al. ${ }^{3}$ demonstrou que os sedimentos em suspensão liberam quantidades significantes de fósforo inorgânico para as águas. Isto poderia explicar parcialmente porque o nitrogênio, e não o fósforo é usualmente limitante na área em estudo.

A dinâmica do ciclo do fósforo também foi avaliada por Aguiar ${ }^{17}$, que estudou a variabilidade sazonal dos teores de fósforo no sistema estuarino de Santos/ São Vicente e descreveu o fósforo particulado como sendo predominante sobre as formas dissolvidas tanto no inverno (maré de quadratura variou de 8,65 a $34,65 \mu \mathrm{mol} \mathrm{L}^{-1} \mathrm{e}$, na maré de sizígia, de 2,71 a 103,86 $\mu \mathrm{mol} \mathrm{L}^{-1}$ ) como no verão (maré de quadratura variou de 6,54 a $35,65 \mu \mathrm{mol} \mathrm{L}^{-1}$ e, na maré de sizígia, de 34,65 a $23,51 \mu \mathrm{mol} \mathrm{L}^{-1}$ ), caracterizando o ambiente como impactado em relação ao fósforo.

No estuário de Tanshui, a concentração de fósforo particulado encontrada por Fang ${ }^{12}$ foi de 0,28 a 9,47 $\mu \mathrm{mol} \mathrm{L}^{-1}$, com uma diminuição da concentração em direção ao mar aberto. Comparando-se os valores descritos para o Canal de Santos e do estuário de Tanshui, com os encontrados no presente estudo, pode-se concluir não haver um incremento acentuado de PTP na Plataforma Continental do Amazonas (para a camada eufótica o mínimo foi de 0,04 e o máximo de $1,78 \mu \mathrm{mol} \mathrm{L}^{-1}$ e, para a camada afótica, o mínimo foi de 
0,01 e máximo de $1,58 \mu \mathrm{mol} \mathrm{L}{ }^{-1}$, Tabela 3 , como os verificados em área impactadas.

Para melhor visualizar e comparar a distribuição do fosfato, PTP e POD nas camadas eufótica e afótica, foram produzidos gráficos de caixa, com valores autoescalonados. Observa-se na Figura 3 uma distribuição assimétrica em ambas as camadas para as formas de fósforo.

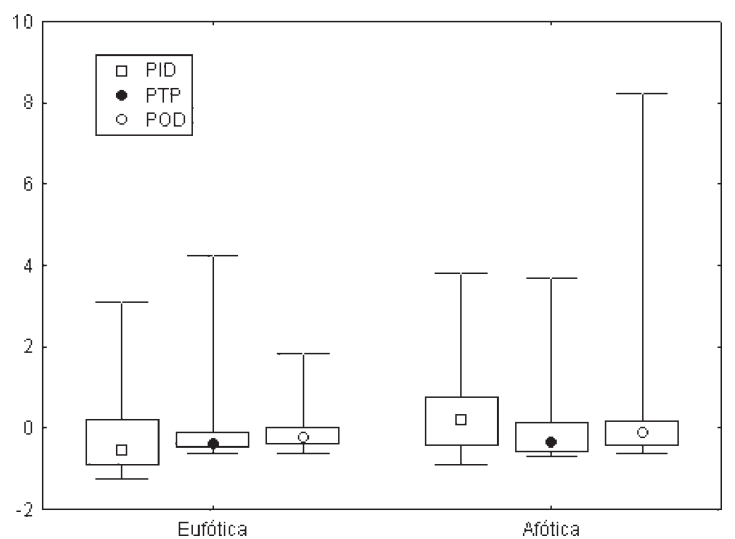

Figura 3. Distribuição dos valores autoescalonados do fosfato (PID), PTP e $P O D$, nas camadas eufótica e afótica

Na camada eufótica observa-se uma cauda superior maior para o PTP, seguido do fosfato e POD. A presença de água fluvial favoreceu o aumento na concentração do material em suspensão. As medianas ficaram próximas aos valores mínimos tanto do PTP quanto do POD. Em relação ao fosfato, a mediana ficou centralizada entre o quartil superior e o quartil inferior, indicando que, apesar do processo fotossintético remover o fósforo em sua forma inorgânica dissolvida, outros processos como a liberação a partir do POD ou do PTP aumenta os valores do fosfato nesta camada (Figura 3).

Na camada afótica, o fosfato registrou a maior mediana, seguido do PTP e POD, indicando que a degradação da matéria orgânica está liberando o fósforo em sua forma inorgânica. Destaca-se também, nesta camada, uma cauda superior bastante acentuada do POD (máximo valor detectado de $1,43 \mu \mathrm{mol} \mathrm{L}{ }^{-1}$, na estação 115 na profundidade de $12 \mathrm{~m}$ ), sendo observado nesta mesma profundidade um valor de PTP de $0,06 \mu \mathrm{mol} \mathrm{L} \mathrm{L}^{-1}$ e de fosfato de $0,89 \mu \mathrm{mol} \mathrm{L} \mathrm{L}^{-1}$.

Ressalta-se que as amplitudes interquatílicas do PTP e POD foram menores, mostrando variações mais limitadas em suas distribuições, o que reforça a idéia de que estas formas de uma forma geral estão sofrendo rápidas transformações dentro do ciclo do fósforo, o que favorece o aumento da concentração do fosfato, tanto na camada eufótica quanto na camada afótica (Figura 3).

De acordo com Day et al. ${ }^{18}$, o fósforo inorgânico dissolvido é assimilado pelas algas e bactérias e o fósforo orgânico particulado pode ser liberado na forma de fosfato ou como fósforo orgânico dissolvido, o qual pode ser decomposto pela ação das bactérias liberando fosfato.

Além do processo acima citado como fonte para o POD, Sanders e Jickell ${ }^{19}$ descreveram que na zona eufótica os nutrientes orgânicos dissolvidos (nitrogênio e fósforo orgânico dissolvido) são produzidos por diferentes mecanismos biológicos, incluindo a exudação pelo fitoplâncton, que pode promover a retenção dos nutrientes nas águas superficiais; "grazing" do zooplâncton; e a solubilização do material orgânico particulado.

Para avaliar a distribuição das formas de fósforo estudadas em função da salinidade foram construídos diagramas de mistura para os dados superficiais. Ressalta-se que os pontos alinhados sobre a linha teórica obedecem à diluição física para os componentes conservativos, enquanto os que apresentam anomalias positivas e negativas sugerem adição e remoção de fósforo. Na Figura 4, observa-se para o fosfato, PTP e POD um comportamento não conservativo.

$\mathrm{Na}$ área em estudo o pH (mínimo de 7,32 e máximo de 8,12, mediana de 7,72) manteve-se sempre alcalino e influenciou muito pouco os processos de adsorção e dessorção do fósforo na coluna de água. Podendo-se verificar a influência das águas oriundas do rio Amazonas na distribuição do fosfato e PTP, com as maiores concentrações localizadas nas estações próximas à costa e uma diminuição em direção ao mar aberto (Figuras 5 e 4b).

A concentração do PTP na coluna de água não apresentou um aumento na região de remoção do fosfato, sugerindo que a adsorção ao material em suspensão não foi um mecanismo importante na região de salinidade a partir de 30 (Figura 4).

O POD apresentou dispersão nos dados superficiais em função da salinidade e as maiores concentrações próximas à foz do rio Amazonas (Figuras 4 e 5).
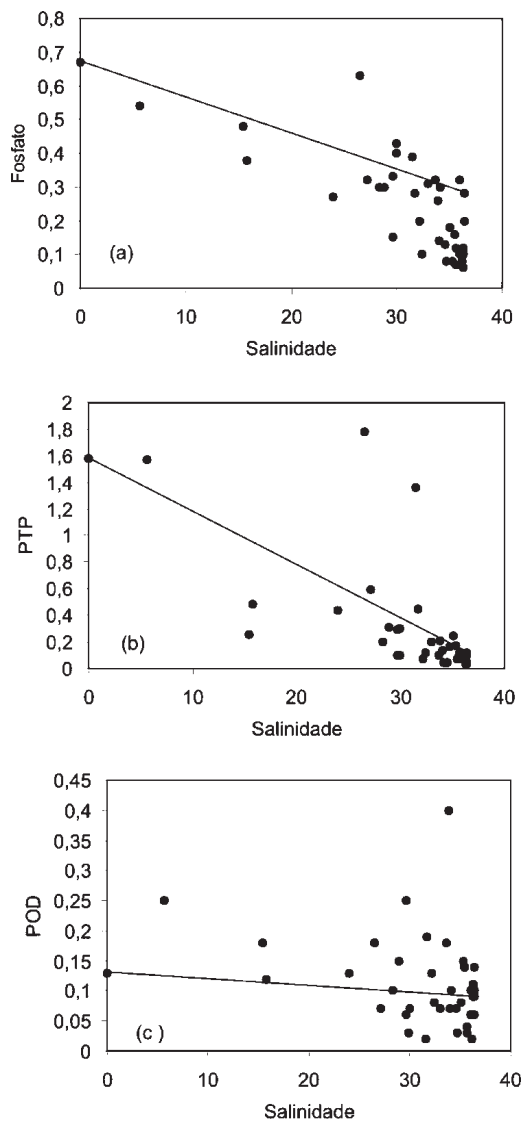

Figura 4. Dados superficiais obtidos durante o período de transição em função da salinidade: (a) fosfato ( $\left.\mu \mathrm{mol} \mathrm{L} L^{-1}\right)$, (b) PTP ( $\left.\mu \mathrm{mol} \mathrm{L} L^{-1}\right),(c)$ POD ( $\left.\mu \mathrm{mol} \mathrm{L} L^{-1}\right)$

\section{CONCLUSÕES}

Os resultados do presente trabalho sugerem que a coluna de água na Plataforma Continental do Amazonas não se encontra impacto pela diferentes formas de fósforo investigado. O POD representou uma pequena fração da forma total dissolvida na coluna de água e o PTP, uma fração importante desse elemento, devido principalmente à liberação para a forma de fosfato. $\mathrm{O} \mathrm{pH}$ alcalino em todas as amostras influenciou muito pouco os processos de adsorção e dessorção do fósforo na coluna de água. Por outro lado, a variação de salinidade existente na Plataforma Continental do Amazonas parece exercer forte influência nos processos de liberação do fósforo para a coluna de água. 

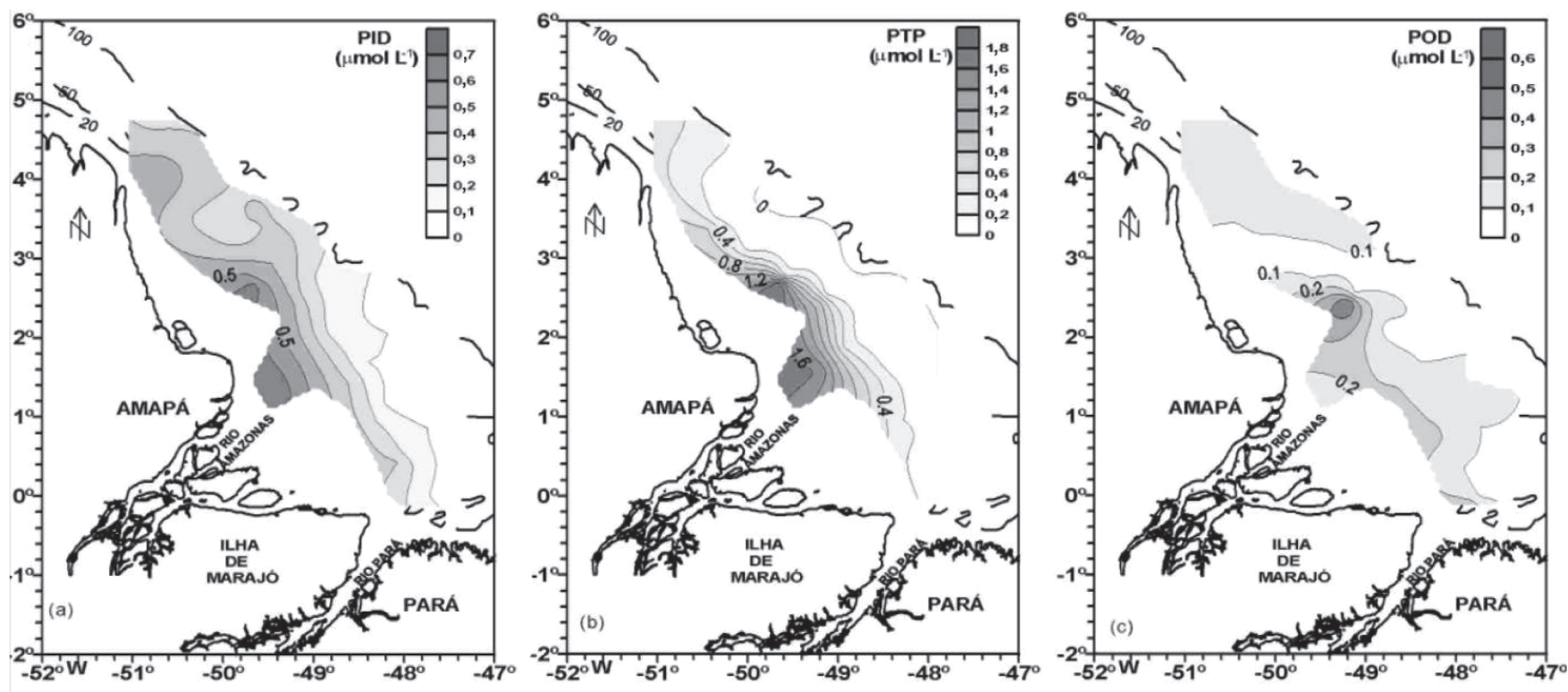

Figura 5. Distribuição superficial do (a) fosfato $\left(\mu \mathrm{mol} L^{-1}\right)$, (b) PTP $\left(\mu \mathrm{mol} L^{-1}\right)$ e (c) POD $\left(\mu \mathrm{mol} L^{-1}\right)$ na área da Plataforma Continental do Amazonas

Na camada eufótica, a descarga fluvial proveniente do rio Amazonas favoreceu a distribuição do fosfato, PTP e POD. Na camada afótica, o percentual do fosfato aumentou, com conseqüência da liberação a partir do POD e do PTP e da ausência do processo fotossintético. O PTP apresentou uma diminuição em seu percentual, o que pode estar relacionado ao processo de deposição.

\section{AGRADECIMENTOS}

Ao Conselho Nacional de Desenvolvimento Científico e Tecnológico $(\mathrm{CNPq})$; à tripulação do Navio Oceanográfico ANTARES (H40), pelo o apoio e atenção recebidos no trabalho de campo, durante a Operação Norte IV (2001); ao Ministério da Defesa (MD)/ Marinha do Brasil (MB); à Comissão Interministerial para os Recursos do Mar (CIRM) e à Diretoria de Hidrografia e Navegação (DHN).

\section{REFERÊNCIAS}

1. Berner, R. A.; Rao, J. L.; Geochim. Cosmochim. Acta 1994, 58, 2333.

2. Kineke, G. C.; Sternberg, R. W.; Trowbridge, J. H.; Geyer, W. R.; Continental Shelf Research 1996, 16, 667.
3. Fox, L. E.; Sager, S. L.; Wofsy, S.; Geochim. Cosmochim. Acta 1986, 50, 783.

4. Fox, L. E.; Geochim. Cosmochim. Acta 1989, 53, 417.

5. Strickland, J. D. H.; Parsons, T. R.; Bulletim Fisheries Research Board Canada 1972, 167, 1.

6. UNESCO; International Oceanographic Table, 1973.

7. Grasshoff, K.; Ehrhardt, M.; Kremling, K.; Methods of seawater analysis. $2^{\text {nd }}$ ed., Verlage Chemie: Florida, 1983

8. Paranhos, R.; Alguns métodos para análise da água, Ed. da UFRJ: Rio de Janeiro, 1996.

9. Teixeira, C.; Bol. Inst. Ocean. São Paulo 1973, 22, 59.

10. Clark, J. F.; Simpson, H. J.; Bopp, R. F.; Deck, B.; Estuar. Coast. Mar. Sci. 1992, 34, 213

11. Flegal, A. R.; Smith, G. J.; Gill, G. A.; Sañudo-Wilhelmy, S. A.; Anderson, L.; Mar. Chem. 1991, 36, 329.

12. Fang, T. H.; Estuar. Coast. Shelf Sci. 2000, 50, 689.

13. Silva, M. A. L.; Calasans, C. F.; Ovalle, A. R. C.; Rezende, C. E.; Braz. Arch. Biol. Technol. 2001, 44, 365.

14. Karl, M. D.; Yanagi, K.; Limnol. Oceanography 1997, 42, 1398.

15. Brockmann, U.; Viehweger, K.; Raabe, T.; Dt. hydrogr. Z 1999, 51, 293.

16. http://gce-lter.marsci.uga.edu/lter/files/pubs/Weston_gwrc_2003.pdf, acessada em Abril 2004.

17. Aguiar, V. M. C.; Dissertação de Mestrado, Universidade de São Paulo, Brasil, 2002

18. Day, J. W.; Hall, C. A. S.; Kemp, W. M.; Arancibia, A. Y.; Estuarine Ecology, Wiley: New York, 1989.

19. Sanders, R.; Jickells, T.; Deep-Sea Res. 2000, 47, 997. 